\title{
Direct imaging of isofrequency contours of guided modes in extremely anisotropic all-
} dielectric metasurface

Pidgayko, Dmitry; Sinev, Ivan S.; Permyakov, Dmitry V.; Sychev, Stanyslav; Heyroth, Frank; Rutckaia, Viktoriia; Schilling, Joerg; Lavrinenko, Andrei V.; Bogdanov, Andrey A.; Samusev, Anton

\section{Published in:}

ACS Photonics

Link to article, DOI:

10.1021/acsphotonics.8b01487

Publication date:

2019

Document Version

Peer reviewed version

Link back to DTU Orbit

Citation (APA):

Pidgayko, D., Sinev, I. S., Permyakov, D. V., Sychev, S., Heyroth, F., Rutckaia, V., Schilling, J., Lavrinenko, A. V., Bogdanov, A. A., \& Samusev, A. (2019). Direct imaging of isofrequency contours of guided modes in extremely anisotropic all-dielectric metasurface. ACS Photonics, 6(2), 510-515.

https://doi.org/10.1021/acsphotonics.8b01487

\section{General rights}

Copyright and moral rights for the publications made accessible in the public portal are retained by the authors and/or other copyright owners and it is a condition of accessing publications that users recognise and abide by the legal requirements associated with these rights.

- Users may download and print one copy of any publication from the public portal for the purpose of private study or research.

- You may not further distribute the material or use it for any profit-making activity or commercial gain

- You may freely distribute the URL identifying the publication in the public portal 


\title{
Direct imaging of isofrequency contours of
}

\section{guided modes in extremely anisotropic all-dielectric metasurface}

\author{
Dmitry Pidgayko, ${ }^{* \dagger}$ Ivan Sinev, ${ }^{\dagger}$ Dmitry Permyakov, ${ }^{\dagger}$ Stanislav Sychev, ${ }^{\dagger}$ \\ Frank Heyroth, Viktoriia Rutckaia," Joerg Schilling," Andrei Lavrinenko, \\ Andrey Bogdanov, ${ }^{\dagger}$ and Anton Samusev ${ }^{\dagger}$ \\ $\dagger$ Department of Nanophotonics and Metamaterials,ITMO University, 197101, \\ Saint-Petersburg, Russia \\ $\ddagger$ Interdisciplinary Center of Material Science, Martin-Luther-University Halle-Wittenberg, \\ Heinrich-Damerow-Strasse 4, 06120 Halle (Saale), Germany \\ - Centre for Innovation Competence SiLi-nano, Martin Luther University \\ Halle-Wittenberg, 06120 Halle, Germany \\ $\S D T U$ Fotonik, Technical University of Denmark, Oersteds pl. 343, DK-2800 Kongens \\ Lyngby, Denmark \\ E-mail: d.pidgayko@metalab.ifmo.ru
}

\begin{abstract}
In this work, we investigate an all-dielectric metasurface based on the silicon on insulator platform for manipulating of guided modes in the visible and near-infrared spectral ranges. We use the Fourier modal method to demonstrate numerically, that guided modes excited in the metasurface support both hyperbolic-like and elliptic dispersion regimes. We implement a back focal plane microscope combined with a high
\end{abstract}


refractive index solid immersion lens to directly image the isofrequency contours of the guided modes (surface waves). Reconstruction of dispersion unambiguously reveals the transition between different dispersion regimes.

\section{Keywords}

All-dielectric metasurface, surface waves, Fourier modal method, isofrequency contours , Fourier microscopy, dispersion reconstruction

Metasurfaces are planar optically thin artificial structures that usually consist of arrays of optically resonant elements. ${ }^{1}$ Preserving the rich functionalities of their bulk counterpart, metamaterials, metasurfaces are cheaper and easier to fabricate, especially for the optical range. $^{2-4}$ At the moment, most of researches in the field of metasurfaces are focused on their application for manipulation of bulk waves, where metasurfaces are used as negative refraction plates, high NA lenses, polarization and phase control devices. ${ }^{5-7}$

Localized modes of the metasurfaces attract special interest. These include surface waves (or guided modes in case of all-dielectric metasurfaces), propagating electromagnetic states $^{8}$ localized perpendicular to the metasurface interface that do not radiate into the farfield. These properties make them very promising for applications in modern optoelectronic and all-optical on-chip signal delivery. In straightforward analogy with metamaterials used for the control over bulk waves, metasurfaces form a flexible platform for manipulating surface waves. ${ }^{9-13}$ In particular, it was shown that a silver grating with the subwavelength period supports the transition from positive to negative refraction, self-collimation and diffractionfree propagation of surface plasmon polaritons in the visible range. ${ }^{14,15}$ Also, hyperbolicallyshaped wavefronts of infrared phonon-polaritons were directly observed in metasurfaces based on nanostructured h-BN. ${ }^{16}$ Whereas this effect in the h-BN structure is observed in the IR spectral range, the main disadvantage of a plasmonic material are high ohmic losses that hinder the application of surface waves (SWs) as on-chip signal carriers in such structures. 
All-dielectric metasurfaces, on the other hand, have significantly lower losses and, therefore, are a viable alternative for plasmonic ones.

Regardless of the type of metasurfaces, their unusual properties have specific fingerprints in the SWs dispersion (the same applies to guided modes) and they can be most conveniently described in terms of isofrequency contours (IFC). IFC is a cross-section of the dispersion at a fixed frequency $\omega\left(k_{x}, k_{y}\right)=\omega_{0}$. Since group velocity $\boldsymbol{V}=\nabla_{\boldsymbol{k}} \omega$ is normal to the IFC, the topology of the latter allows one to draw conclusions about the peculiarities of SW propagation. For example, the transition from positive to negative refraction of a surface plasmon-polariton between flat and nanostructured silver is a straightforward consequence of IFCs transformation from elliptic (with negative curvature) to hyperbolic shape (with positive curvature). ${ }^{17,18}$ Diffraction-free propagation of surface waves arises at zero curvature of IFCs. Finally, hyperbolically shaped wavefronts are an explicit consequence of hyperbolic IFCs. ${ }^{8} 14$ However, despite the fact that the majority of the effects observed in Refs. ${ }^{15,16}$ are explained through transition from elliptic to hyperbolic regimes, the IFCs of such SWs were not directly visualized. In other works, methods based on additional fabrication techniques, ${ }^{19,20}$ samples with strong disorder,${ }^{21}$ resonance-enhanced photon scattering ${ }^{22}$ were used for this purpose. Recently, we have introduced a method that provides direct imaging of IFCs of localized states based on back focal plane (BFP) microscopy with a solid immersion lens (SIL). The method has been successfully used to visualize the IFCs of waveguide modes of a planar isotropic silicon waveguide. ${ }^{23}$

In this work, we propose a design of an extremely anisotropic all-dielectric SOI-based metasurface for the visible and near-IR spectral ranges which supports elliptic and hyperboliclike regimes of IFCs of guided modes (GMs) . We directly visualize the IFCs using a back focal plane microscopy setup with a solid immersion lens. Finally, we reconstruct the complete dispersion relation $\omega\left(k_{x}, k_{y}\right)$ of GMs supported by the metasurface experimentally and numerically. 


\section{Setup}

Since the dispersion law of GMs resides under the light line of the ambient media, they cannot be directly excited from free space due to the momentum mismatch. ${ }^{8}$ The missing momentum required for the GM excitation can be gained, for example, through scattering of light on nanoparticles ${ }^{24,25}$ or periodic gratings. ${ }^{26,27}$ However, such configurations usually require near-field techniques to measure the fields of GMs and, therefore, they are not suitable for direct imaging of IFCs. Another option is to use the attenuated total internal reflection (TIR) with prism coupling (also known as Otto configuration), which is commonly used for characterization the dispersion relation. ${ }^{28-30}$ However, standard Otto reflection setups only allow for measurements within a certain plane of incidence and they are, thus, hardly applicable for characterization of anisotropic structures due to the dependence of the dispersion on the in-plane direction.

Here, we use a setup that combines back focal plane microscopy with the Otto excitation configuration based on a solid immersion lens. The experimental setup is shown in Figure 1 (a). For excitation we employ a supercontinuum laser source Fianium WhiteLase SC400-6, combined with an acousto-optic Fianium AOTF filter, which gives a spectral line with a width of $4 \mathrm{~nm}$ tunable in the broad range from visible to near-IR $(\lambda=650-1100$ $\mathrm{nm}$ ). In experiment, linearly polarized light is focused with a high-NA objective (Mitutoyo, M Plan Apo HR, 100x, 0.9 NA) on the flat surface of a hemispherical ZnSe solid immersion lens (SIL) with refractive index $n \approx 2.5$ in the near-IR. ${ }^{31}$ 


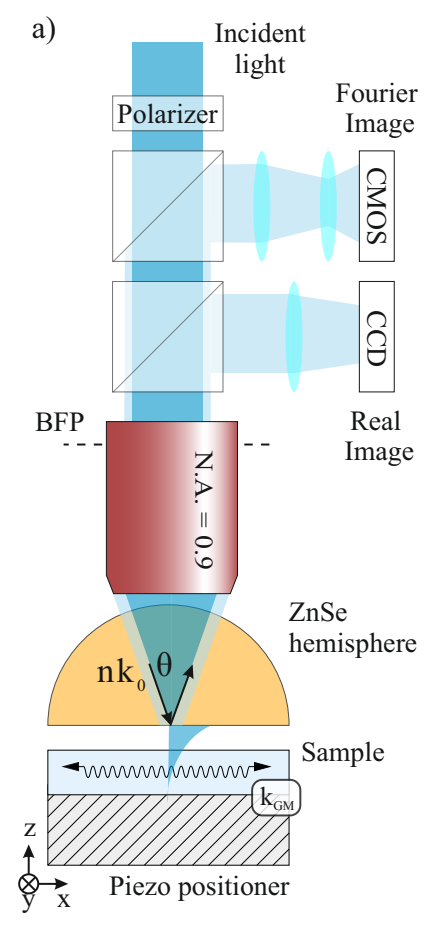

b)

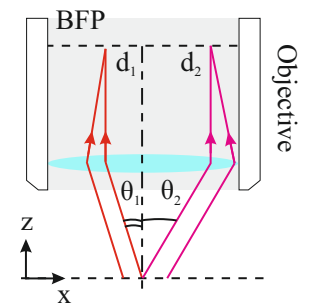

c)

Longitudinal polarization

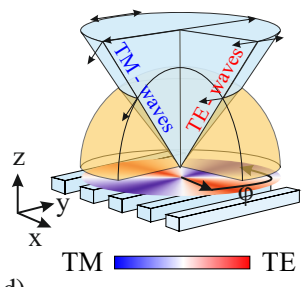

d)

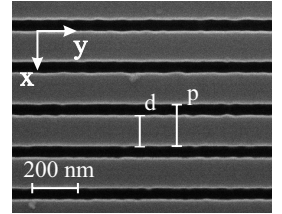

Figure 1: (a) Experimental setup layout. (b) Scheme of back focal plane imaging: angles are transformed to coordinates in the Fourier plane. For simplicity, the ZnSe hemisphere is not included. (c) Polarization distribution in the BFP for the case of polarization along the metasurface bars (longitudinal polarization) (d) SEM image of the sample.

In this configuration, the maximum attainable wavevector of the GMs measured in the units of free-space wavevector $\mathrm{k}_{0}$ excitation equals to

$$
\frac{k_{G M}}{k_{0}}=n N A \approx 2.25
$$

where $\mathrm{k}_{G M}$ is a wave vector of guided modes. Excitation of the GM is manifested as a dip in reflectivity at a particular angle. The depth of the dip that represents the GM coupling efficiency in attenuated TIR configuration depends on the gap between the hemisphere and the sample, and is controlled with high precision by a piezo positioner ( $\mathrm{S} 1$ supplementary). In the Fourier image channel of the setup (see Figure 1a) we use a combination of lenses to project the image of the BFP on the CMOS camera matrix. Since the angular spectrum of the radiation in the real plane is transformed into the spatial distribution in the BFP 
(see Figure 1b), pronounced reflection dips represent IFCs. In this way, the SIL - BFP experimental setup facilitates excitation of GMs and simultaneous visualization of their IFCs in a broad spectral range.

\section{Results and discussions}

We consider an anisotropic all-dielectric metasurface represented by a dense grating composed of subwavelength rectangular slabs made of crystalline silicon (See Figure 1d) with period $p=180 \mathrm{~nm}$, width $d=125 \mathrm{~nm}$ and height $h=70 \mathrm{~nm}$. The metasurface was fabricated on the SOI substrate using e-beam lithography followed by reactive ion etching. We apply the Fourier modal method (FMM) widely used for modeling of the optical properties of gratings ${ }^{32-34}$ to simulate the reflectivity from the metasurface at the BFP in Otto geometry and its dependence on the wavelength and polarization (Figure 2 i-l column ). As mentioned earlier, such reflection maps reproduce the IFCs of GMs . We specified the parameters of the metasurface in such a way that elliptic and hyperbolic-like regimes are supported only for TE polarized waves . 

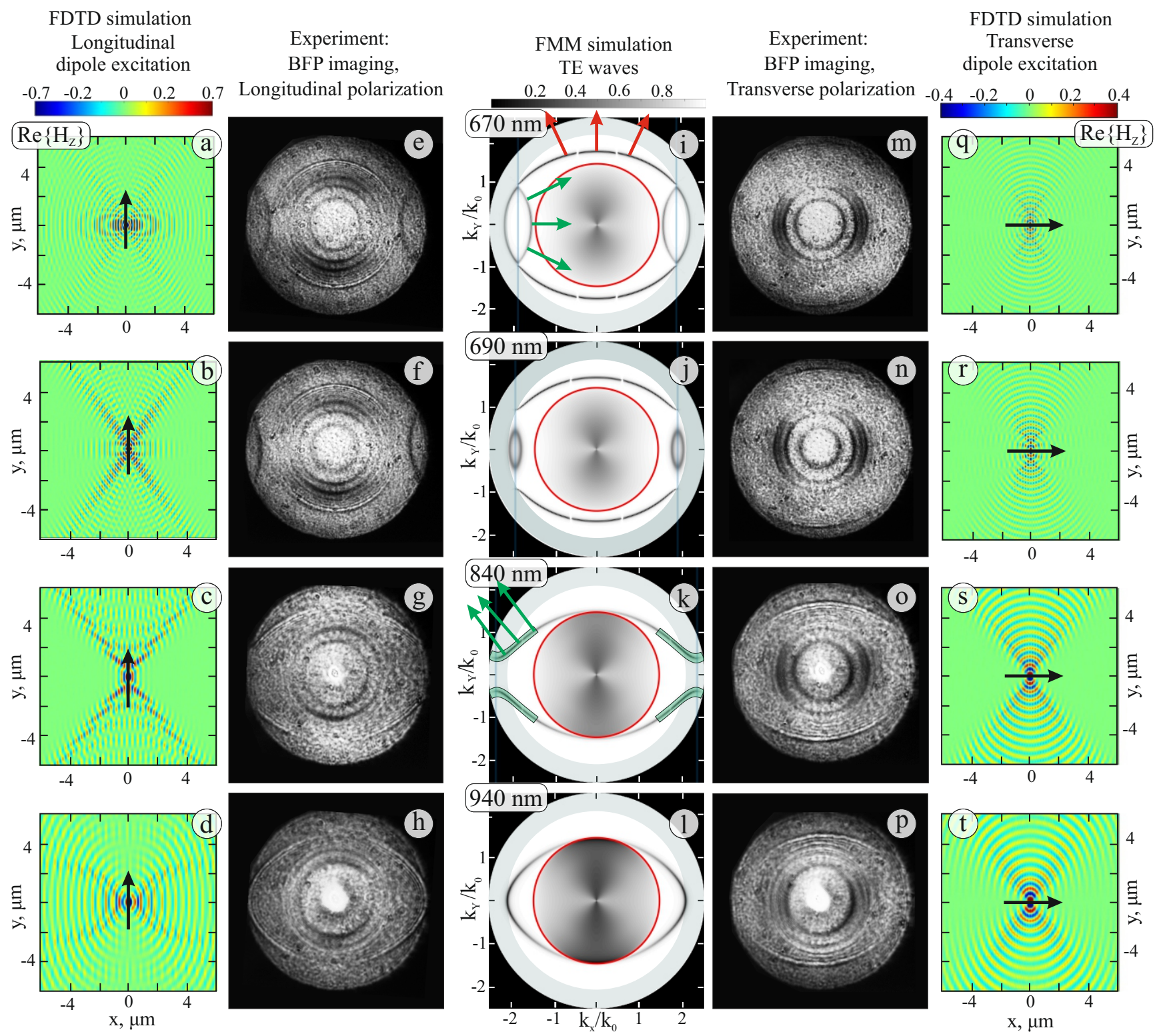

Figure 2: (a-d, q-t): Simulated maps for $\operatorname{Re}\left(H_{z}\right)$ of the modes excited by a point electric dipole in the plane of metasurface, oriented along (a-d) and across ( $\mathrm{q}-\mathrm{t}$ ) the bars. (e-h, m-p): Experimental reflectivity maps under longitudinal (e-h) and transverse (m-p) polarization. (i-l): FMM calculated reflectivity maps in BFP for TE GMs. Red circles correspond to TIR boundaries between $\mathrm{SiO}_{2}$ and $\mathrm{ZnSe}$. Shaded area is not available for observation in the experiment according to Eq.(1). Vertical blue lines represent edges of the normalized first Brillouin zone $\frac{2 \pi}{d k_{0}}$. The arrows show the direction of the group velocity.

In the simulated images (Figure 2 i-l column ), the red circle designates the boundary of TIR between $\mathrm{ZnSe}$ and $\mathrm{SiO}_{2}$ (buffer layer). This circle is experimentally observed when the SIL is maximally pushed towards the sample (S1 supplementary). Boundary of the light-blue 
shaded area limits the experimentally available wave vectors [ see Eq. (1)]. The grey lines between the red circle and the shaded area are actually for the IFCs of TE GMs supported by the metasurface.

To compare simulated reflection maps with the experimental results (Figure 2 e-h and m-p columns ), it is necessary to take into account an important feature of the setup - nonuniform polarization distribution imaged in the BFP under the uniformly linear incident beam polarization, as illustrated in Figure 1c. When the incident light has longitudinal polarization with respect to the bars, the angular distribution of polarization at the $\mathrm{BFP}|P\rangle$ can be written as:

$$
|P\rangle=\cos ^{2}(\varphi)|T E\rangle+\sin ^{2}(\varphi)|T M\rangle
$$

where $\varphi$ is the azimutal angle counted from the $\mathrm{x}$ - axis, $|T E\rangle$ and $|T M\rangle$ are linearly polarized waves of the correspondent polarization. Therefore, for transverse or longitudinal incident polarizations full TE IFCs are only partially imaged in the BFP (compare e-h and m-p columns in Figure 2). Combining them together, one can obtain full contours for TE GMs. Over against, FMM allows to simulate IFCs for GMs with polarization, given by Eq. (2). We present these simulation in Supplementary (S1, S2, S3) where, in addition to polarization, the thickness of the buffer layer is taken into account (S1), which results in Newton's rings observation. Here we ignore them for simplicity. It should be also noted that while the numerical aperture of the system was expected to be 2.25 [Eq. (1)] from the measurements it was estimated to be 2.19. We associate this with a non-ideal spherical shape of the SIL and possible deviations of the refractive index of the ZnSe prism from the tabular values. ${ }^{31}$

We illustrate the process of efficient wave front shaping in our metasurface with Lumerical FDTD. Namely, we model the field distribution of the waves excited by a point electric dipole located in the plane of the metasurface. In accordance with the geometry of the experiment, we carried out simulations for the dipole oriented both along and across the bars. Note that 
in such an orientation the polarization of GMs is defined by Eq. (2). We clarify the relation between IFCs and field profiles in terms of the group velocity, which is responsible for the propagation direction of GMs. The group velocity is normal to the IFC, with its positive direction towards the IFC with higher frequency (see the arrows in Figure 2, i and k ).

We start our interpretation with the case of the transverse incident wave polarization and, accordingly, transverse dipole orientation. The BFP images (Figure 2 m-p column ) reveal characteristic IFC regimes for the studied metasurface. By sketching the direction of the group velocity (red arrows in Figure 2 i ) one can see that these contours define divergent convex wavefronts and negative curvature of contours. The FDTD simulations (Figure 2, q-t column ) confirm this prediction.

The case of longitudinal dipole excitation and longitudinal incident wave polarization reveals notably different regimes of GMs propagation. At $\lambda=670 \mathrm{~nm}$ the contours have hyperbolic-like branches with positive curvature along the transverse direction (Figure 2 e-h column ). These are formed when elliptic contours intersect the edges of the first Brillouin zone (vertical blue lines on Figure 2 i-l column), and are folded back inside the first Brillouin zone. With the increase of the wavelength, the edge of the normalized Brillouin zone $\left(\frac{\pi}{d k_{0}}\right)$ moves towards larger normalized wavevectors, whereas the IFC squeezes up to shorter wavevectors (Figure $2 \mathrm{j}$ ). In contrast the case of transverse polarization, the group velocity for these states is directed towards the centre of the first Brillouin zone ( green arrows in Figure $2 \mathrm{k}$ ). Therefore, branches effectively possess negative curvature (similarly to elliptic regime), leading to convex divergent radiation pattern(Figure 2 a,b ).

With the increase of the wavelength, the convex branches vanish and within a certain spectral region GMs attain a stop band in the $k_{x}$ direction (Figure $2 \mathrm{k}$ ). Note that branches which correspond to experimental IFC excited by longitudinal polarization at 840 nm (highlighted by green) possess normals with approximately collinear direction. That feature clearly manifests itself in the field simulation, where we see a characteristic cruciform wave front of propagating GM . 
Finally, in Figure $2 \mathrm{~h}$ the observed contour regains the elliptic shape, similarly to the transverse case. Despite seeming simplicity, it is necessary to take into account periodicity in the x-direction, in which GMs obey dispersion not only of the first Brillouin zone, but also of the adjacent one. In the FDTD simulation (Figure $2 \mathrm{~d}$ ), it manifests as interference of states with the wave vectors from the first and second the Brillouin zones.

To confirm that simulated fields straightforwardly relate to the IFCs excited by linear (longitudinal and transverse) polarization, we performed the Fourier transform for $H_{z}$ (See supplementary S2 and S3), which agrees nicely with both experimental and simulated IFCs.

Ultimately, using all available data, we recover complete GMs dispersion $\omega\left(k_{x}, k_{y}\right)$. For this, we derive an isofrequency curve for each excitation wavelength in the whole measured range $\lambda=670-1070 \mathrm{~nm}$ and combine them as shown in Figure 3(a).

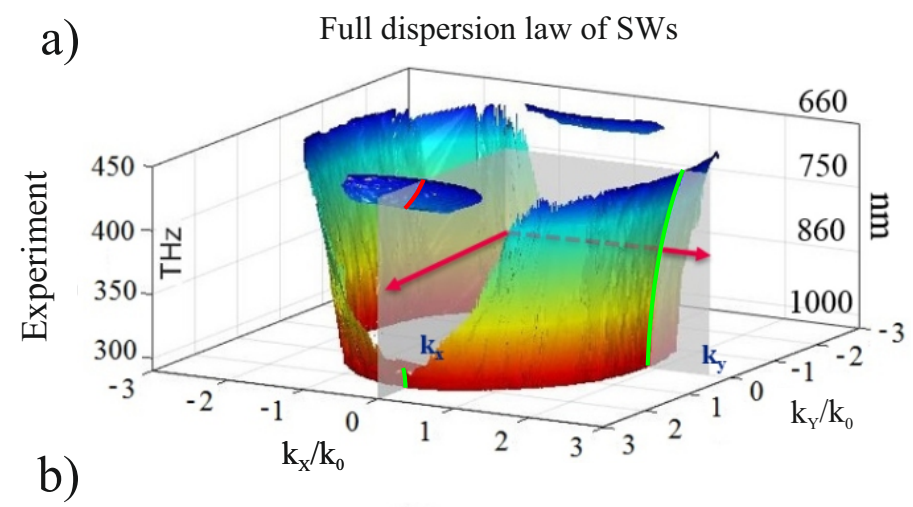

c)
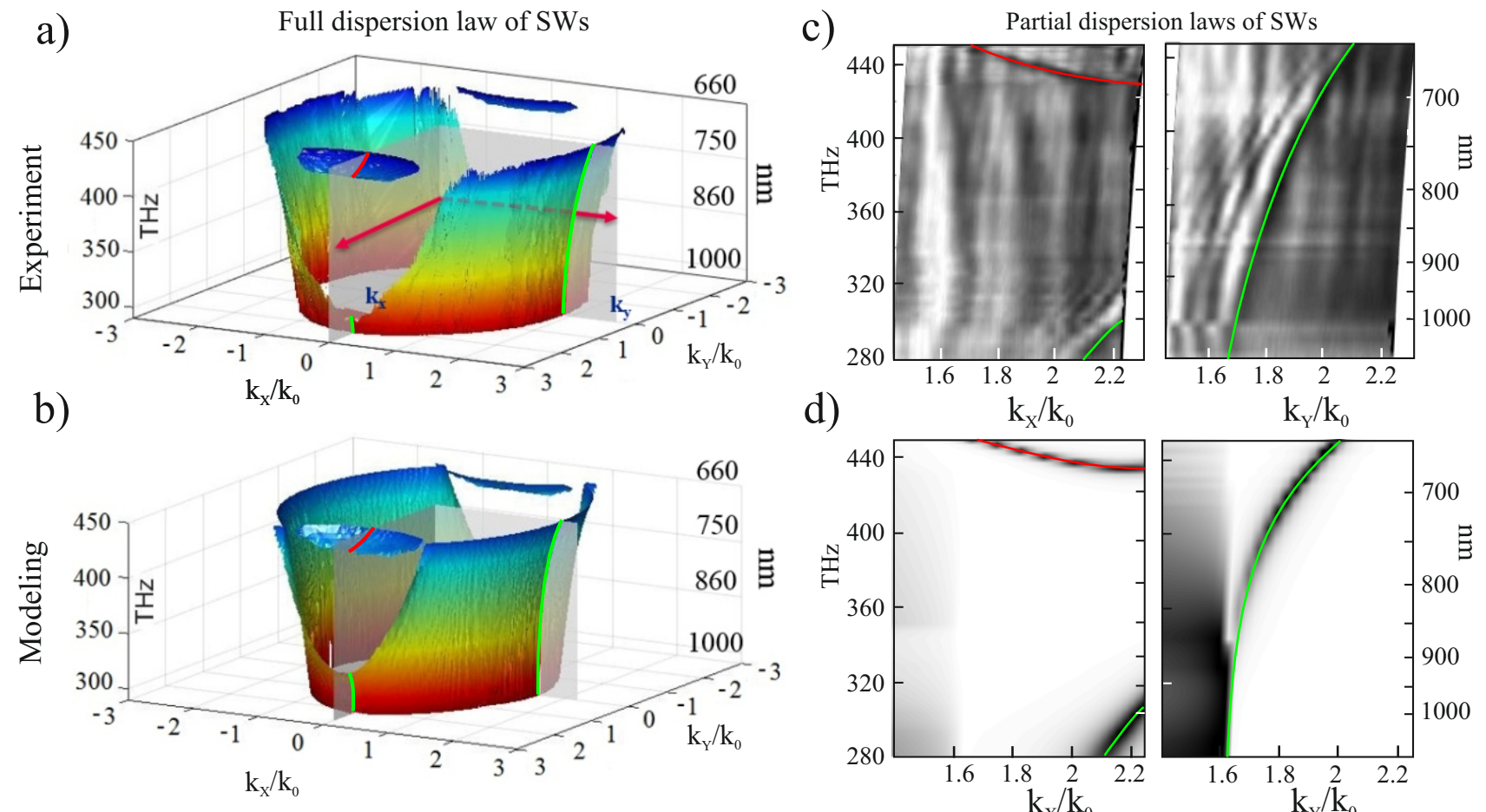

d)

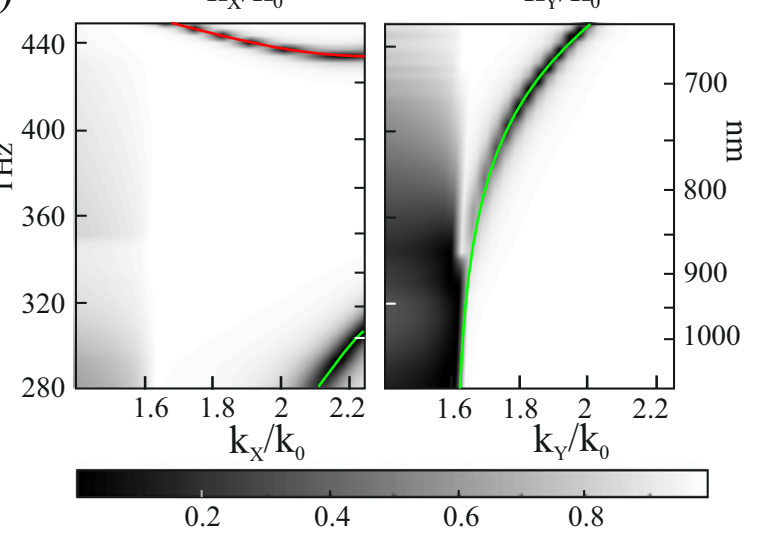

Figure 3: (a,b) Reconstructed experimental and numerical dispersions $\omega\left(k_{x}, k_{y}\right)$. Color added for clarity. The red cross-section curve represents the modes with hyperbolic-like contours, the green curves represent the modes with conventional elliptic dispersion. (c,d) Experimental and simulated cross sections for reflectivity along the $\mathrm{x}$ and $\mathrm{y}$ directions. 
Vertical cross-sections to this surface are conventional dispersion curves along particular directions. For example, for $\mathrm{X}$ and $\mathrm{Y}$ directions the cross sections of stacked IFCs are plotted in Figure $3(\mathrm{c}, \mathrm{d})$. In the longitudinal $(\mathrm{Y})$ direction we see a trivial continuous waveguidelike dispersion, but in the transverse (X) direction, branches for elliptic and hyperbolic-like regimes are observed with a wide stop band between them.

\section{Conclusion}

We have studied dispersion properties of optical GMs supported by a strongly anisotropic all-dielectric metasurface. The nanostructured array composed of closely-packed silicon bars on a silica buffer layer was designed to support hyperbolic-like and elliptic dispersion regimes. In the experiment, the IFCs of GMs were visualized by means of back focal plane microscopy. The use of a hemispherical solid immersion lens allowed to drastically enhance the numerical aperture of the system up to $\mathrm{NA} \approx 2.19$ and gain access to strongly localized states. Using FDTD simulations, we illustrated multiple dispersion regimes depending on the wavelength and polarization of the incident wave. Finally, joining the whole set of the experimental data

we have the full dispersion $\omega\left(k_{x}, k_{y}\right)$ of guided modes propagating along the metasurface in all directions.

\section{Acknowledgement}

This work was supported by the Ministry of Education and Science of the Russian Federation (Zadanie No.3.8891.2017/8.9).

\section{Supporting information description}

Experimental and FMM back focal plane images for different air gap sizes, expanded experimental and FMM back focal plane images, FDTD fields simulation in real and Fourier space 
for longitudinal and transverse polarization (dipole orientation) respectively.

\section{References}

(1) Glybovski, S. B.; Tretyakov, S. A.; Belov, P. A.; Kivshar, Y. S.; Simovski, C. R. Metasurfaces: From microwaves to visible. Physics Reports 2016, 634, 1-72.

(2) Kildishev, A. V.; Boltasseva, A.; Shalaev, V. M. Planar photonics with metasurfaces. Science 2013, 339, 1232009.

(3) Wu, C.; Arju, N.; Kelp, G.; Fan, J. A.; Dominguez, J.; Gonzales, E.; Tutuc, E.; Brener, I.; Shvets, G. Spectrally selective chiral silicon metasurfaces based on infrared Fano resonances. Nature communications 2014, 5, 3892.

(4) Zhan, A.; Colburn, S.; Trivedi, R.; Fryett, T. K.; Dodson, C. M.; Majumdar, A. Lowcontrast dielectric metasurface optics. ACS Photonics 2016, 3, 209-214.

(5) Yu, N.; Genevet, P.; Kats, M. A.; Aieta, F.; Tetienne, J.-P.; Capasso, F.; Gaburro, Z. Light propagation with phase discontinuities: generalized laws of reflection and refraction. science 2011, 334, 333-337.

(6) Yu, N.; Capasso, F. Flat optics with designer metasurfaces. Nature materials 2014, 13, $139 \hat{a} \mathrm{~A} S ̧ 150$.

(7) Aieta, F.; Genevet, P.; Kats, M. A.; Yu, N.; Blanchard, R.; Gaburro, Z.; Capasso, F. Aberration-free ultrathin flat lenses and axicons at telecom wavelengths based on plasmonic metasurfaces. Nano letters 2012, 12, 4932-4936.

(8) Takayama, O.; Bogdanov, A.; Lavrinenko, A. V. Photonic surface waves on metamaterial interfaces. Journal of Physics: Condensed Matter 2017, 29, 463001. 
(9) Samusev, A.; Mukhin, I.; Malureanu, R.; Takayama, O.; Permyakov, D.; Sinev, I.; Baranov, D.; Yermakov, O.; Iorsh, I.; Bogdanov, A.; A.Lavrinenko, Time-Resolved Luminescence Nanothermometry with Nitrogen-Vacancy Centers in Nanodiamonds. Optics Express 2017, 25, 32631-32639.

(10) Rance, H. J.; Constant, T. J.; Hibbins, A. P.; Sambles, J. R. Surface waves at microwave frequencies excited on a zigzag metasurface. Physical Review B 2012, 86, 125144.

(11) Shitrit, N.; Yulevich, I.; Maguid, E.; Ozeri, D.; Veksler, D.; Kleiner, V.; Hasman, E. Spin-optical metamaterial route to spin-controlled photonics. Science 2013, 340, 724726.

(12) Martini, E.; Mencagli, M.; Maci, S. Metasurface transformation for surface wave control. Phil. Trans. R. Soc. A 2015, 373, 20140355.

(13) Li, Y. B.; Cai, B. G.; Wan, X.; Cui, T. J. Diffraction-free surface waves by metasurfaces. Optics letters 2014, 39, 5888-5891.

(14) Liu, Y.; Zhang, X. Metasurfaces for manipulating surface plasmons. Applied Physics Letters 2013, 103, 141101.

(15) High, A. A.; Devlin, R. C.; Dibos, A.; Polking, M.; Wild, D. S.; Perczel, J.; de Leon, N. P.; Lukin, M. D.; Park, H. Visible-frequency hyperbolic metasurface. Nature 2015, 522, $192 \mathrm{a} \breve{A} S ̧ 196$.

(16) Li, P.; Dolado, I.; Alfaro-Mozaz, F. J.; Casanova, F.; Hueso, L. E.; Liu, S.; Edgar, J. H.; Nikitin, A. Y.; Velez, S.; Hillenbrand, R. Infrared hyperbolic metasurface based on nanostructured van der Waals materials. Science 2018, 359, 892-896.

(17) Ferrari, L.; Wu, C.; Lepage, D.; Zhang, X.; Liu, Z. Hyperbolic metamaterials and their applications. Progress in Quantum Electronics 2015, 40, 1-40. 
(18) Belov, P. Backward waves and negative refraction in uniaxial dielectrics with negative dielectric permittivity along the anisotropy axis. Microwave and Optical Technology Letters 2003, 37, 259-263.

(19) Giannattasio, A.; Barnes, W. L. Direct observation of surface plasmon-polariton dispersion. Optics express 2005, 13, 428-434.

(20) Le Thomas, N.; Houdré, R.; Beggs, D.; Krauss, T. Fourier space imaging of light localization at a photonic band-edge located below the light cone. Physical Review B 2009, 79, 033305.

(21) Shi, L.; Yin, H.; Zhu, X.; Liu, X.; Zi, J. Direct observation of iso-frequency contour of surface modes in defective photonic crystals in real space. Applied Physics Letters 2010, 97, 251111.

(22) Regan, E. C.; Igarashi, Y.; Zhen, B.; Kaminer, I.; Hsu, C. W.; Shen, Y.; Joannopoulos, J. D.; Soljačić, M. Direct imaging of isofrequency contours in photonic structures. Science advances 2016, 2, e1601591.

(23) Permyakov, D. V.; SinevS., I. S.; Sychev, K.; Gudovskikh, A. S.; Bogdanov, A. A.; Lavrinenko, A. V.; Samusev, A. K. Visualization of Isofrequency Contours of Strongly Localized Waveguide Modes in Planar Dielectric Structures. JETP Letters 2018, 107, 10-14.

(24) Sinev, I.; Iorsh, I.; Bogdanov, A.; Permyakov, D.; Komissarenko, F.; Mukhin, I.; Samusev, A.; Valuckas, V.; Kuznetsov, A. I.; Luk'yanchuk, B. S.; Miroshnichenko, A. E.; Kivshar, Y. S. Polarization control over electric and magnetic dipole resonances of dielectric nanoparticles on metallic films. Laser $\mathscr{E}$ Photonics Reviews 2016, 10, 799-806.

(25) Sinev, I.; Bogdanov, A.; Komissarenko, F.; Petrov, M.; Frizyuk, K.; Makarov, S.; Mukhin, I.; Samusev, A.; Lavrinenko, A.; Iorsh, I. Demultiplexing surface waves with silicon nanoantennas. AIP Conference Proceedings. 2017; p 030035. 
(26) Worthing, P.; Barnes, W. L. Efficient coupling of surface plasmon polaritons to radiation using a bi-grating. Applied Physics Letters 2001, 79, 3035-3037.

(27) Taillaert, D.; Bienstman, P.; Baets, R. Compact efficient broadband grating coupler for silicon-on-insulator waveguides. Optics letters 2004, 29, 2749-2751.

(28) Ballarini, M.; Frascella, F.; Enrico, E.; Mandracci, P.; De Leo, N.; Michelotti, F.; Giorgis, F.; Descrovi, E. Bloch surface waves-controlled fluorescence emission: coupling into nanometer-sized polymeric waveguides. Applied Physics Letters 2012, 100, 39.

(29) Zhang, D. G.; Yuan, X.; Bouhelier, A. Direct image of surface-plasmon-coupled emission by leakage radiation microscopy. Applied optics 2010, 49, 875-879.

(30) Frisbie, S.; Chesnutt, C.; Holtz, M.; Krishnan, A.; De Peralta, L. G.; Bernussi, A. Image formation in wide-field microscopes based on leakage of surface plasmon-coupled fluorescence. IEEE Photonics Journal 2009, 1, 153-162.

(31) Marple, D. Refractive index of ZnSe, ZnTe, and CdTe. Journal of Applied Physics 1964, 35, 539-542.

(32) Li, L. New formulation of the Fourier modal method for crossed surface-relief gratings. JOSA A 1997, 14, 2758-2767.

(33) Li, L. Fourier modal method for crossed anisotropic gratings with arbitrary permittivity and permeability tensors. Journal of Optics A: Pure and Applied Optics 2003, 5, 345.

(34) Li, L. Mathematical modeling in optical science; SIAM, 2001; pp 111-139. 


\section{For Table of Contents Use Only}

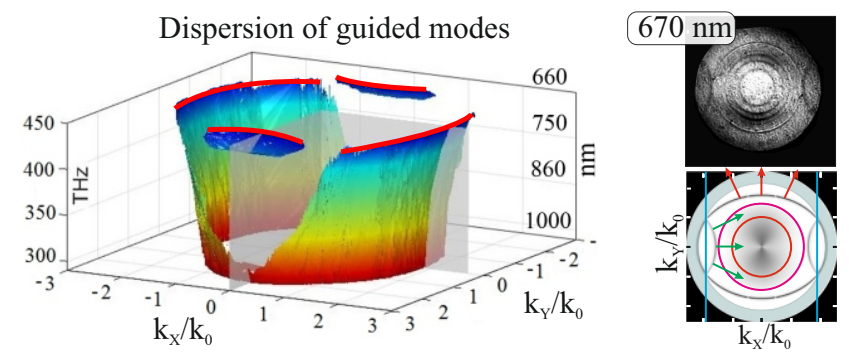

Manuscript title: Direct imaging of isofrequency contours guided modes in extremely anisotropic all-dielectric metasurface.

Authors: Dmitry Pidgayko, Ivan Sinev, Dmitry Permyakov, Stanislav Sychev, Frank Heyroth, Viktoriia Rutckaia, Joerg Schilling, Andrei Lavrinenko, Andrey Bogdanov and Anton Samusev.

Synopsis: Our work is devoted to experimental observation of isofrequency contours of guided modes in all-dielectic metasurface. Using back focal plane microscopy with solid immersion lens. We investigate SOI - based metasurface represented by subwavelength alldielectric grating, that supports both hyperbolic-like end elliptic dispersion regimes. Combining all experimental data we reconstruct the full dispersion relation $\omega\left(k_{x}, k_{y}\right)$. 